\title{
CONSTRUCTION AND APPLICATION OF THE CLASSIFICATION SCHEME OF DYNAMIC RISK MEASURES ESTIMATING
}

\author{
Nataliia Zrazhevska \\ Department of Mathematical Methods of Systems Analysis \\ National Technical University of Ukraine "Igor Sikorsky Kyiv Polytechnic Institute", \\ 37 Peremohy str., Kyiv, Ukraine, 03056 \\ natasha.zrazhevska@gmail.com
}

\begin{abstract}
The most popular methods for dynamic risk measures - Value-at-Risk (VaR) and Conditional VaR (CVaR) estimating were analyzed, description and comparative analysis of the methods were fulfilled, recommendations on the use were given. Results of the research were presented in the form of a classification scheme of dynamic risk measures estimating that facilitates the choice of an estimation method. The GARCH-based models of dynamic risk measures VaR and CVaR evaluation for artificially generated series and two time series of log return on a daily basis of the most well-known Asian stock indexes Nikkey225 Stock Index and CSI30 were constructed to illustrate the effectiveness of the proposed scheme. A qualitative analysis of the proposed models was conducted. To analyze the quality of the dynamic VaR estimations the Cupets test and the Cristoffersen test were used. For CVaR estimations the V-test was used as quality test. The tests results confirm the high quality of obtained estimations. The proposed classification scheme of dynamic risk measures VaR and CVaR estimating may be useful for risk managers of different financial institutions.
\end{abstract}

Keywords: Dynamic Value at Risk, dynamic Conditional Value at Risk, heteroscedastic model, Nikkey225 Stock Index, CSI300 Index.

\section{Introduction}

Extreme price movements in the financial markets are rather rare but they have a great influence on the financial and political processes in the world. Events such as the stock market crash on Wall Street in October 1987, the bankruptcy of the US investment bank Lehman Brothers in 2008, caused significant market volatility and the instability of financial institutions. This, in turn, stressed the relevance of evaluation of possible financial risks. VaR and CVaR (also known as ES - Expected Shortfall) are among the most popular risk measures. The VaR concept was proposed in [1], and in 1999 risk measure CVaR was introduced [2].

Nowadays, there is a great number of works, dedicated to the properties of these risk measures and their comparative analysis [3, 4]. Article [5] presents the review of the known methods for CVaR estimating, the examples of evaluation of these risk measures for specific data can be found in [6-11]. The large number of methods, that are actively appearing, leads to the necessity to fulfill their systematization and classification using the methods of system analysis. Such approach simplifies the process of selecting the optimal way for solving the problem for risk management [12].

Building econometric models, risk managers often use a standard indicator - return of some asset which can be understood as: $\mathrm{X}_{\mathrm{t}}=\mathrm{P}_{\mathrm{t}}-\mathrm{P}_{\mathrm{t}-1}$, where $\mathrm{P}_{\mathrm{t}}$ is the price of an asset at time $t$, or simple return $R_{t}=P_{t} / P_{t-1}-1$, or $\log$ return $X_{t}=\ln \left(P_{t} / P_{t-1}\right)=\ln \left(R_{t}+1\right)$. Log return is very popular because in this case there is no reference to the specific terms of money and this definition allows to scale a sharp price changes.

If it is necessary to describe the profitability for the time period that is multiple to the basic period with the multiplicity $k$ we will mean the value of profitability as $X_{t}^{k}=\ln P_{t} / P_{t-k}=\ln \left(R_{t}^{k}+1\right)$.

While understanding the time series as a realization of a stochastic process, it is essential to define a temporary shift for getting the values of random variable. In the econometric applications, this period can vary from 1 minute to 1 year. In our article this option is not being considered. We assume that the successive values (sorted by time) of the time series with the increased by 1 index differ by a single period. 
Formalizing asset as a random variable, depending on the time, we get a time series $\left\{X_{t}, t \in Z\right\}$. Time series can be described by different stochastic models $[13,14]$ and so we get different methods for the risk measures estimations. In this article we analyze the most popular time series models used to obtain VaR and CVaR estimates, give their classification (Fig. 1), and offer the guidance on their usage. Methods that represent different approaches are tested on artificially simulated and real data. Comparative analysis of the results is presented.

Analysis of the main methods for VaR and CVaR estimating allowed to develop the structural scheme for the choice of the method for dynamic risk measures VaR and CVaR and estimating according to the research objectives and the characteristics of the analyzed data (Fig. 2).

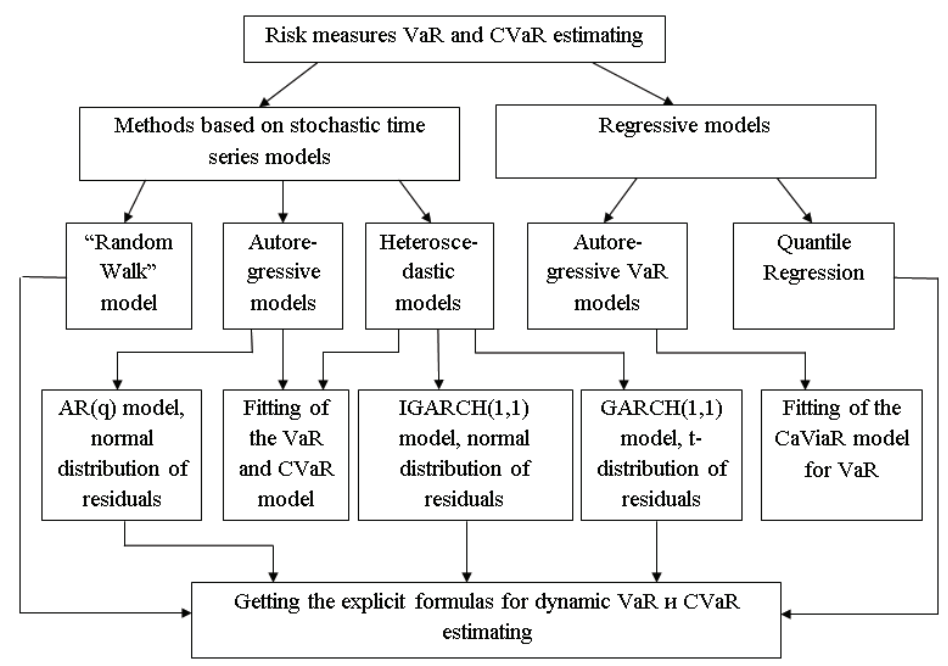

Fig. 1. The classification scheme for the choice of the method for dynamic risk measures VaR and CVaR estimating

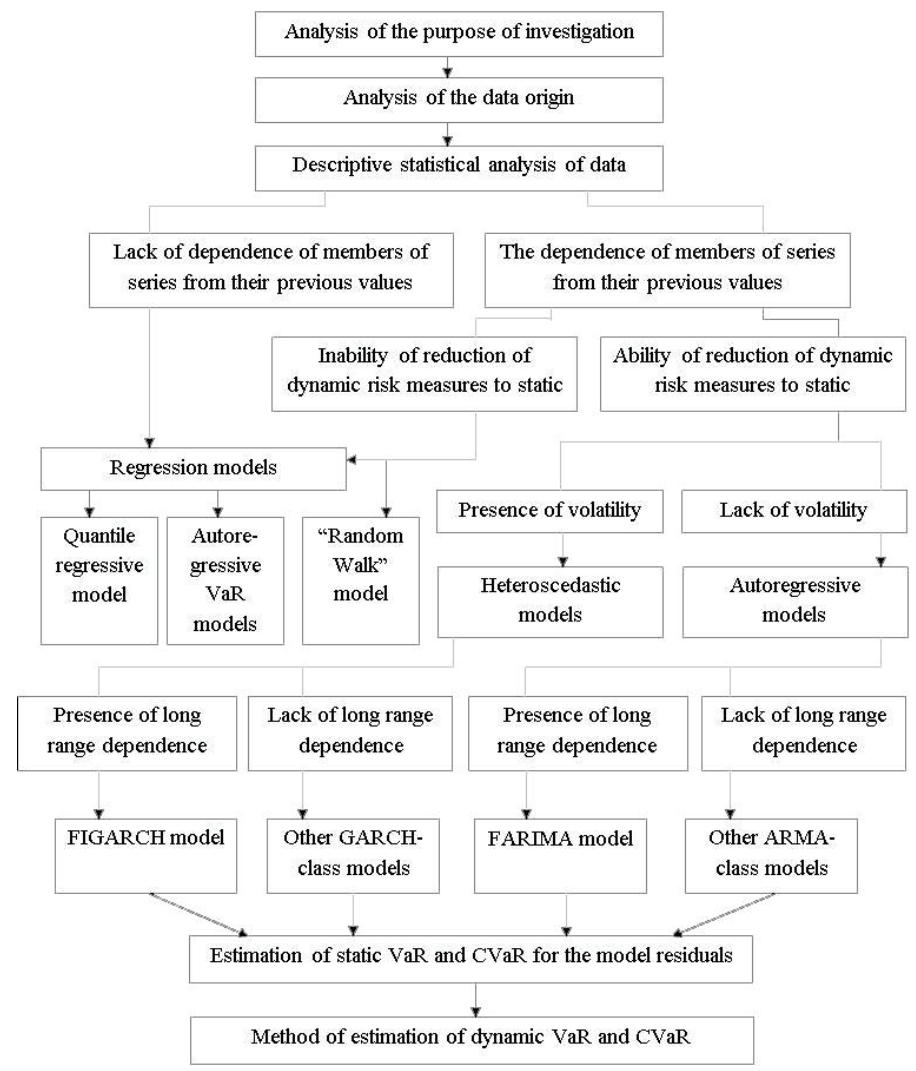

Fig. 2. The structural scheme of selection of dynamic risk measures estimation 
The brief description of the methods and an example of using this scheme is given in the following sections. Methods that represent different approaches are tested on artificially simulated and real data; a comparative analysis of the results is presented.

\section{Materials and Methods}

We consider the continuously distributed random variable $\left\{X_{t}, t \in Z\right\}$ with finite mean, defined on the probability space $\left\{\Omega, \Psi_{t}, \mathrm{P}_{t}\right\}$, where $\Psi_{t}$ is the information set containing all available at the time $t$ information about the time series. For a fixed confidence level $\alpha$ risk measures $\operatorname{VaR}_{\alpha}^{\tilde{\mathrm{t}}}$ and $\mathrm{CVaR}_{\alpha}^{\tilde{\mathrm{t}}}$ are defined as $[13,15]$ :

$$
\begin{gathered}
\operatorname{VaR}_{\alpha}^{\mathrm{t}}(\mathrm{t})=\inf \left\{\mathrm{x} \in \mathrm{R} \mid \mathrm{P}_{\mathrm{t}}\left[\mathrm{X}_{\mathrm{t}} \leq \mathrm{x}\right] \geq \alpha\right\} \operatorname{VaR}_{\alpha}^{\mathrm{t}}(\tilde{\mathrm{t}})=\inf \left\{\mathrm{x} \in \mathrm{R} \mid \mathrm{P}_{\mathrm{t}}\left[\mathrm{X}_{\tilde{\mathrm{t}}} \leq \mathrm{x}\right] \geq \alpha\right\}, \\
\mathrm{CVaR}_{\alpha}^{\mathrm{t}}(\mathrm{t})=\mathrm{E}_{\Psi_{\mathrm{t}}}\left[\mathrm{X}_{\mathrm{t}} \mid \mathrm{X}_{\mathrm{t}} \geq \operatorname{VaR}_{\alpha}^{\mathrm{t}}(\mathrm{t})\right] \operatorname{CVaR}_{\alpha}^{\mathrm{t}}(\tilde{\mathrm{t}})=\mathrm{E}_{\Psi_{\mathrm{t}}}\left[\mathrm{X}_{\tilde{\mathrm{t}}} \mid \mathrm{X}_{\tilde{\mathrm{t}}} \geq \operatorname{VaR}_{\alpha}^{\mathrm{t}}(\tilde{\mathrm{t}})\right] .
\end{gathered}
$$

By $\mathrm{E}_{\Psi_{t}}[\cdot]$ we denote expectation with respect to $\Psi_{t}$.

$\operatorname{VaR}_{\alpha, \mathrm{k}}^{\mathrm{t}}, \mathrm{CVaR}_{\alpha, \mathrm{k}}^{\mathrm{t}}$ define the risk measures at time $\mathrm{t}+\mathrm{k}$, defined on the information set $\Psi_{\mathrm{t}}$ :

$$
\operatorname{VaR}_{\alpha, \mathrm{k}}^{\mathrm{t}}=\operatorname{VaR}_{\alpha}^{\mathrm{t}}(\mathrm{t}+\mathrm{k}), \quad \mathrm{CVaR}_{\alpha, \mathrm{k}}^{\mathrm{t}}=\mathrm{CVaR}_{\alpha}^{\mathrm{t}}(\mathrm{t}+\mathrm{k})
$$

\section{1. Analysis of Methods of Estimating Based on Stochastic Time Series Models}

In this chapter we consider the approaches to risk measures estimating on the basis of commonly used time series models [13-15].

\section{"Random Walk" Model}

The time series $\left\{X_{t}\right\}$ follows the model "random walk" (RW) process if $X_{t}=X_{t-1}+\varepsilon_{t}$, where $\left\{\varepsilon_{\mathrm{t}}\right\} \sim \mathrm{N}\left(\mu, \sigma^{2}\right)$ (independent normal random variables with mean $\mu$ and variance $\sigma^{2}$ ).

Obviously, the following estimates take place [15]:

$\hat{\mu}=\frac{1}{n} \sum_{i=1}^{n} \varepsilon_{i}, \hat{\sigma}=\sqrt{\frac{1}{n-1} \sum_{i=1}^{n}\left(\varepsilon_{i}-\hat{\mu}\right)^{2}}$. Scaling rule (the square-root-of-time rule) can be performed to the model. If $X_{t}^{k}=X_{t-1}^{k}+\varepsilon_{t}^{k}, \varepsilon_{t} \sim k \mu+\sqrt{k}\left(\varepsilon_{t}-\mu\right)$, then $\hat{\mu}^{k}=k \hat{\mu}, \hat{\sigma}^{k}=\sqrt{k} \hat{\sigma}$ and for a given confidence level $\alpha$ and time period $k$ risk measures estimations can be written as:

$$
\widehat{\operatorname{VaR}}_{\alpha, \mathrm{k}}^{\mathrm{t}}=\hat{\mu}^{\mathrm{k}}+\hat{\sigma}^{\mathrm{k}} \mathrm{x}^{(\alpha)}, \widehat{\operatorname{CVaR}}_{\alpha, \mathrm{k}}^{\mathrm{t}}=\hat{\mu}^{\mathrm{k}}+\frac{\left(\hat{\sigma}^{\mathrm{k}}\right)^{2}}{2} \frac{\Phi\left(\mathrm{x}^{(\alpha)}-\hat{\sigma}^{\mathrm{k}}\right)}{1-\alpha},
$$

where $\Phi(\cdot)$ denotes the standard normal ccumulative distribution function (cdf), $x^{(\alpha)}$ its $\alpha$ quantile.

The advantages of the RW model are its simplicity and the availability of ready-made formulas for risk measures estimating. In addition, it is the most known model of non-stationary time series ( 1 is a root of the characteristic equation). The disadvantage of the model is the fact that its residuals are considered to be normally distributed, that is a significant limitation in the modeling of real financial time series. Residuals distribution approximates to the normal distribution with the sample period increasing, so RW model is used for modeling of financial instruments with a large (about a year) reporting period [13].

\section{Autoregressive Models for Risk Measures Estimating}

Autoregressive model of order $\mathrm{q}-\mathrm{AR}(\mathrm{q})$ assumes that the value of the series at the time $\mathrm{t}$ is determined by its q past values [13]: $X_{t}=\sum_{i=1}^{q} a_{i} X_{t-i}+\varepsilon_{t} \quad X_{t}=\sum_{i=1}^{q} a_{i} X_{t-i}+\varepsilon_{t}$, where coefficients $a_{t}$ satisfy the stationarity condition $\sum_{\mathrm{i}=1}^{\mathrm{q}}\left|\mathrm{a}_{\mathrm{i}}\right|<1,\left\{\varepsilon_{\mathrm{t}}\right\}$ are independent and identically distributed random variables (iid). If innovations $\left\{\varepsilon_{\mathrm{t}}\right\}$ are normally distributed: $\left\{\varepsilon_{\mathrm{t}}\right\} \sim \mathrm{N}\left(\mu_{0}+\mu_{1} \mathrm{t}, \sigma^{2}\right)$, the article [15] presents the explicit formulas for VaR and CVaR estimating. 
Let's consider the time series $\left\{\overline{X_{t}}\right\}$, where $\overline{X_{t}}=X_{t}-\mu t, \mu=\mu_{1} /\left(1-\sum_{i=1}^{q} a_{i}\right)$. Then the $\operatorname{AR}(q)$ model for $\left\{\overline{X_{t}}\right\}$ can be written as: $\overline{X_{t}}=\sum_{i=1}^{q} a_{i} \overline{X_{t-i}}+\overline{\varepsilon_{t}},\left\{\overline{\varepsilon_{t}}\right\} \sim N\left(0, \sigma^{2}\right)$.

Let $\hat{\mathrm{q}}, \hat{\mathrm{a}}_{\mathrm{i}}, \hat{\sigma}$ denote the maximum likelihood estimates, then for risk measures estimating formulas (1) can be used, where $\hat{\mu}^{\mathrm{k}}=\mathrm{k} \hat{\mu}+\hat{\mathrm{m}}, \quad \hat{\sigma}^{\mathrm{k}}=\hat{\sigma} \sqrt{\sum_{\mathrm{j}=0}^{\mathrm{k}-1} \delta_{j}^{2}}, \quad \hat{\mu}=\frac{\mathrm{X}_{\mathrm{n}}-\mathrm{X}_{0}}{\mathrm{n}}, \quad \hat{\mathrm{m}}=\tilde{X}_{\mathrm{t}+\mathrm{k}}-\tilde{X}_{\mathrm{t}}, \quad \delta_{0}=1$, $\delta_{\mathrm{j}}=\sum_{\mathrm{i}=1}^{\mathrm{j}} \hat{\mathrm{a}}_{\mathrm{i}} \mathrm{I}\{\mathrm{i}=\mathrm{j}\} \quad\left(\mathrm{I}\{\}\right.$ denotes the indicator function), $\hat{\mathrm{a}}_{\mathrm{i}}=0$ for $\mathrm{i}>\hat{\mathrm{q}}$. The sequence $\left\{\tilde{\mathrm{X}}_{\mathrm{t}+\mathrm{k}}\right\}$ can be find recursively: $\tilde{X}_{t+j}=\sum_{i=1}^{\hat{q}} \hat{a}_{i} \tilde{X}_{t+j-i}, j=\overline{1, k}, \tilde{X}_{u}=\bar{X}_{u}$ for $\mathrm{u} \leq \mathrm{t}$.

In the moving average model $\mathrm{MA}(\mathrm{p})$ the values of time series are determined by the weighted values of the model innovations $[13,14]: X_{t}=\sum_{j=1}^{p} b_{j} \varepsilon_{t-j}+\varepsilon_{t}$.

The model $\operatorname{ARMA}(\mathrm{q}, \mathrm{p})$ is the generalization of the models $\operatorname{AR}(\mathrm{q})$ and $\operatorname{MA}(\mathrm{p})$ : $\mathrm{X}_{\mathrm{t}}=\sum_{\mathrm{i}=1}^{\mathrm{q}} \mathrm{a}_{\mathrm{i}} \mathrm{X}_{\mathrm{t}-\mathrm{i}}+\sum_{\mathrm{j}=1}^{\mathrm{p}} \mathrm{b}_{\mathrm{j}} \varepsilon_{\mathrm{t}-\mathrm{j}}+\varepsilon_{\mathrm{t}}$. The information criteria (AIC, BIC) [13] are often used to find the orders $\mathrm{p}$, q of the model and maximum likelihood method can be used to estimate the model parameters $a_{i}, i=\overline{1, q}, b_{j}, j=\overline{1, p}$.

There are many tests to verify the iid property of the residuals, see, for example, [16]. In this study the BDS test [16] and the variance ratio test [17] were used.

Note, that there are many modifications of the model ARMA(q, p), for example, a model FARIMA(q, d, p) that takes into account the effect of the long-range dependence.

ARMA class models are homoscedastic parametric models $\left(\sigma_{t}=\sigma=c o n s t\right)$. Financial series frequently exhibit volatility clustering, so heteroscedastic models such as GARCH models are often used for their modeling.

Heteroscedastic Models for Risk Measures Estimating

Let's suppose that the time series $\left\{X_{t}, t \in Z\right\}$ is a stochastic process, that is,

$$
X_{t}=\mu_{t}+\varepsilon_{t}=\mu_{t}+\sigma_{t} Z_{t}
$$

where conditional mean $\mu_{t}$ and variation $\sigma_{t}$ are defined in the information space $\Psi_{t},\left\{Z_{t}\right\} \sim F_{t}(0,1)$ (independent, identically distributed random variables with a conditional distribution function $F_{t}(0,1)$ ). $\mathrm{Z}$ is a random variable with the same distribution as any random variable from $Z_{t}$. Then $[13,18]$ :

$$
\begin{aligned}
& \operatorname{VaR}_{\alpha, k}^{\mathrm{t}}=\mu_{\mathrm{t}+\mathrm{k}}+\mathrm{F}^{-1}(\alpha) \sigma_{\mathrm{t}+\mathrm{k}}=\mu_{\mathrm{t}+\mathrm{k}}+\mathrm{VaR}_{\alpha}(\mathrm{Z}) \sigma_{\mathrm{t}+\mathrm{k}}, \\
& \mathrm{CVaR}_{\alpha, \mathrm{k}}^{\mathrm{t}}=\mu_{\mathrm{t}+\mathrm{k}}+\mathrm{F}^{-1}(\alpha) \sigma_{\mathrm{t}+\mathrm{k}}=\mu_{\mathrm{t}+\mathrm{k}}+\mathrm{CVaR}_{\alpha}(\mathrm{Z}) \sigma_{\mathrm{t}+\mathrm{k}} .
\end{aligned}
$$

Thus, for $\operatorname{VaR}_{\alpha, k}^{\mathrm{t}}$ and $\mathrm{CVaR}_{\alpha, \mathrm{k}}^{\mathrm{t}}$ estimation it is necessary to estimate $\mu_{\mathrm{t}+\mathrm{k}}, \sigma_{\mathrm{t}+\mathrm{k}}$ and to get the estimations of risk measures $\operatorname{VaR}_{\alpha}(Z)$ and $\mathrm{CVaR}_{\alpha}(\mathrm{Z})$ for one random variable. Depending on the methods used for estimation we have different approaches for getting $\operatorname{VaR}_{\alpha, k}^{\mathrm{t}}$ and $\mathrm{CVaR}_{\alpha, \mathrm{k}}^{\mathrm{t}}$. Methods for $\operatorname{VaR}_{\alpha}(Z)$ and $\mathrm{CVaR}_{\alpha}(\mathrm{Z})$ estimating are described in detail in [19] for example. To estimate the conditional variation, let's consider the following models.

One of the most classic variance estimating models is the MA model [6]:

$$
\hat{\sigma}_{t}^{2}=\frac{1}{m-1} \sum_{j=1}^{m}\left(X_{t-j}-\hat{\mu}_{t}^{m}\right)^{2}, \text { where } \hat{\mu}_{t}^{m}=\frac{1}{m} \sum_{j=1}^{m} X_{t-j}, m \in N
$$

Popular in the applications the GARCH(q, p) model can be written as [13, 14]:

$$
X_{t}=\mu_{t}+\varepsilon_{t}=\mu_{t}+\sigma_{t} Z_{t}, \sigma_{t}^{2}=a_{0}+\sum_{i=1}^{q} a_{i} \varepsilon_{t-i}^{2}+\sum_{j=1}^{p} b_{j} \sigma_{t-j}^{2},
$$


where $0<a_{0}<\infty, a_{i} \geq 0, i=\overline{1, q}, \quad b_{i} \geq 0, i=\overline{1, p}, \quad \sum_{i=1}^{\max (p, q)}\left(a_{i}+b_{i}\right)<1$. With the use of the shift operator $\mathrm{LX}_{\mathrm{t}}=\mathrm{X}_{\mathrm{t}-1}$, (4) can be rewritten as:

$$
(1-\alpha(\mathrm{L})-\beta(\mathrm{L})) \varepsilon_{\mathrm{t}}^{2}=\mathrm{a}_{\mathrm{o}}+(1-\beta(\mathrm{L})) \mathrm{v}_{\mathrm{t}} \text {, where } \alpha(\mathrm{L})=\sum_{\mathrm{i}=1}^{\mathrm{q}} \mathrm{a}_{\mathrm{i}} \mathrm{L}^{\mathrm{i}}, \beta(\mathrm{L})=\sum_{\mathrm{j}=1}^{\mathrm{p}} \mathrm{b}_{\mathrm{j}} \mathrm{L}^{\mathrm{j}}, \mathrm{v}_{\mathrm{t}} \equiv \varepsilon_{\mathrm{t}}^{2}-\sigma_{\mathrm{t}}^{2}-\text { iid. }
$$

Model for which $\alpha(1)+\beta(1)=1$ is $\operatorname{IGARCH}(\mathrm{q}, \mathrm{p})$ : $\phi(\mathrm{L})(1-\mathrm{L}) \varepsilon_{\mathrm{t}}^{2}=\mathrm{a}_{0}+(1-\beta(\mathrm{L})) \mathrm{v}_{\mathrm{t}}$, where the operator $\phi(L) \equiv(1-\alpha(L)-\beta(L))(1-L)^{-1}$ has the order $\max (q, p)-1$. Note that the IG$\mathrm{ARCH}(1,1)$ model is the RiskMetrics model or EWMA model.

Introducing the operator of fractional difference

$$
(1-L)^{d}=\sum_{j=1}^{\infty} \frac{\Gamma(j-d)}{\Gamma(-d) \Gamma(j+1)}(-L)^{j}
$$

where $\Gamma(\cdot)$ is the gamma function, we have the model $\operatorname{FIGARCH}(\mathrm{q}, \mathrm{d}, \mathrm{p}): \phi(\mathrm{L})(1-\mathrm{L})^{\mathrm{d}} \varepsilon_{\mathrm{t}}^{2}=$ $=\mathrm{a}_{0}+(1-\beta(\mathrm{L})) \mathrm{v}_{\mathrm{t}}$. If $\mathrm{d} \in(0,1 / 2)$ the model with normal distributed innovations describes time series with long-range dependence with the Hurst parameter $\mathrm{H}=1 / 2+\mathrm{d}$. We can find the orders $\mathrm{p}, \mathrm{q}$ and parameters $a_{i}, i=\overline{1, q}$ and $b_{i}, i=\overline{1, p}$ using the same methods as we use for ARMA(q,p) model. The methods for d estimation are described, for example, in [20].

The considered approach of risk measures estimating is widely used in research. For example, in [6] the authors use GARCH, IGARCH models for $\sigma_{t}$ estimating and the method of historical simulation and EVT to get risk measures for one random variable. In [7] the IGARCH and FIGARCH models are used. The authors consider the following distributions of model residuals: the normal distribution, the generalized Pareto distribution (GPD), the normal inverse Gaussian distribution. The model DJ RGARCH with $\mathrm{t}$ - distribution for volatility movement is used for VaR evaluation in [9]. Estimation using FIGARCH model with $\mathrm{t}$ - distribution for residuals is considered in [8]. In some works parametric estimation methods are combined with the non-parametric methods for risk measures estimation for one random variable. For example, [10] presents the filtration historical method in combination with the historical method. Kernel evaluation of the probability distribution function (pdf) of the random variable is used in [11].

In some cases, the application of this approach allows to obtain explicit formulas for $\mathrm{VaR}_{\alpha, \mathrm{k}}^{\mathrm{t}}$ and $\mathrm{CVaR}_{\alpha, \mathrm{k}}^{\mathrm{t}}$ evaluation. The paper [15] considers the $\operatorname{GARCH}(1,1)$ model where the innovations are assumed to be Student-t distributed with v degrees of freedom:

$$
\begin{gathered}
X_{t}=X_{t-1}+\mu+\varepsilon_{t}=X_{t-1}+\mu+\sigma_{t} Z_{t}, \\
\sigma_{t}^{2}=a_{0}+a_{1} \varepsilon_{t-1}^{2}+b_{1} \sigma_{t-1}^{2} .
\end{gathered}
$$

The stationarity conditions $0<a_{0}<\infty, a_{1} \geq 0, b_{1} \geq 0, a_{1}+b_{1}<1$ are assumed to be fulfilled. Then risk measures can be estimated by:

$$
\widehat{\operatorname{VaR}}_{\alpha, \mathrm{k}}^{\mathrm{t}}=\hat{\mu}^{\mathrm{k}}+\hat{\sigma}^{\mathrm{k}} \mathrm{x}_{\hat{\mathrm{v}}^{\mathrm{k}}}^{(\alpha)}, \widehat{\operatorname{CVaR}}_{\alpha, \mathrm{k}}^{\mathrm{t}}=\frac{1}{\alpha} \int_{0}^{\alpha}\left(\hat{\mu}^{\mathrm{k}}+\hat{\sigma}^{\mathrm{k}} \mathrm{x}_{\hat{\mathrm{v}}^{\mathrm{k}}}^{(\mathrm{q})}\right) \mathrm{dq}
$$

where $\mathrm{x}_{\mathrm{v}}^{(\alpha)}, \mathrm{x}_{\hat{\mathrm{v}}}^{(\alpha)}$ denotes the $\alpha$ quantile of a Student-t distributed random variable with mean zero and variance one, $\hat{\mu}, \hat{a}_{0}, \hat{a}_{1}, \hat{b}_{1}$ are maximum likelihood estimates, $\hat{\mu}^{\mathrm{k}}=k \hat{\mu}$. The integral can be evaluated numerically. The estimation procedure for the parameter $\mathrm{v}^{\mathrm{k}}, \hat{v}^{\mathrm{k}}$ can be found in [15]. The following algorithm is used to evaluate $\sigma^{\mathrm{k}}=\sigma(\mathrm{t}, \mathrm{t}), \hat{\sigma}^{\mathrm{k}}=\hat{\sigma}(\mathrm{t}, \mathrm{t})$ :

$$
\begin{gathered}
\sigma^{2}\left(\mathrm{t}^{*}, \mathrm{t}\right)=\mathrm{a}_{0}+\mathrm{a}_{1}\left(\varepsilon_{\mathrm{t}^{*}}^{\mathrm{k}}-\mu^{\mathrm{k}}\right)^{2}+\mathrm{b}_{1} \sigma^{2}\left(\mathrm{t}^{*}-\mathrm{k}, \mathrm{t}\right){ }^{\prime} \sigma^{2}(\mathrm{t}-\mathrm{nk}, \mathrm{t})=\frac{\mathrm{k}}{\mathrm{nk}-1} \sum_{\mathrm{i}=1}^{\mathrm{nk}-1}\left(\mathrm{X}_{\mathrm{t}-\mathrm{i}}-\mu\right)^{2}, \\
\mathrm{t}^{*}=\mathrm{t}-(\mathrm{n}-1) \mathrm{k}, \ldots, \mathrm{t}-\mathrm{k}, \mathrm{t} .
\end{gathered}
$$




$$
\begin{gathered}
\hat{\sigma}^{2}\left(t^{*}, t\right)=\hat{a}_{0}+\hat{a}_{1}\left(\varepsilon_{t^{*}}^{k}-\hat{\mu}^{k}\right)^{2}+\hat{b}_{1} \hat{\sigma}^{2}\left(t^{*}-k, t\right), \hat{\sigma}^{2}(t-n k, t)=\frac{k}{n k-1} \sum_{i=0}^{n k-1}\left(X_{t-i}-\hat{\mu}\right)^{2}, \\
t^{*}=t-(n-1) k, \ldots, t-k, t
\end{gathered}
$$

This model is well-suited for reproducing the heteroscedastic behavior of the conditional volatility of time series. The model is used for estimating the risk measures on term from 1 to 10 days. For the period more than 15 days this method gives overstated results. For the period of more than 30 days the stationarity conditions don't take place [15].

One of the simplest and the most common model is the Riskmetrics model (RM model) $[5,21]$. Consider the model:

$$
X_{t}=\sigma_{t} Z_{t}, \quad \sigma_{t}^{2}=(1-\lambda) \sum_{j=1}^{t-1} \lambda^{j-1} X_{t-j}^{2}, \quad \lambda \in(0,1),\left\{Z_{t}\right\}^{\text {iid }} \sim N(0,1)
$$

For large enough $\mathrm{t}(7)$ is well approximated by the model IGARCH$(1,1): \sigma_{t}^{2}=\lambda \sigma_{t-1}^{2}+(1-\lambda) X_{t-1}^{2}$. Then risk measures can be estimated by:

$$
\widehat{\operatorname{VaR}}_{\alpha, \mathrm{k}}^{\mathrm{t}}=\sqrt{\mathrm{k}} \Phi^{-1}(\alpha) \sigma_{\mathrm{t}}, \widehat{\operatorname{CVaR}}_{\alpha, \mathrm{k}}^{\mathrm{t}}=\frac{\sqrt{\mathrm{k}}}{\alpha}\left(\Phi^{-1}(\alpha)\right) \sigma_{\mathrm{t}},
$$

where $\varphi(\cdot)$ denotes the standard normal pdf.

The models GARCH(1,1) and RM are very popular because they give simple and understandable to economists formulas for risk measures estimating. Even if the time series is not adequately described by these models, the analytical formulas allow assessing trends of risk changes with sufficient reliability. Comparative analysis of the models is given in [22]. In the work both models were tested at 31 Stock Exchange of the countries of G7 and Asian and the following practical recommendations were made. RM model should be used for risk estimating for small, emerging markets and large enough confidence level $(\alpha>0.95)$. The GARCH model works well with large, developed markets.

\section{2. Analysis of Methods of Quantile Estimation Methods for VaR and CVaR}

Quantile estimation provides a nonparametric approach to VaR and CVaR calculation [13]. Approach does not require any assumptions about the form of cdf, except that the distribution continues to hold within the considered period. From VaR definition we have that this value is the quantile of the cdf. Unlike all previous methods that modeling the distribution function, quantile approach models the conditional quantile. There are two types of quantile methods - regressive and autoregressive.

\section{Quantile Regression}

We consider one-period risk measures. To estimate $\operatorname{VaR}_{\alpha}^{t}$ we have to get the conditional quantile estimation: $x^{(\alpha)} \mid \Psi_{t-1}$. Let's suppose that we have the linear regression [23]: $X_{t}=\bar{Z}_{t} \bar{\beta}$, where $\bar{Z}_{t}$ is a vector of predictors from $\Psi_{t-1}, \bar{\beta}$ is a vector of parameters. Then we can estimate the conditional quantile as:

$$
\hat{\mathrm{x}}^{(\alpha)} \mid \Psi_{\mathrm{t}-1} \equiv \inf \left\{\bar{Z}_{\mathrm{t}}^{\mathrm{T}} \bar{\beta} \mid \bar{\beta}=\arg \min \Phi(\bar{\beta})\right\}
$$

where $\Phi(\bar{\beta})=\sum_{t=1}^{\mathrm{n}} \lambda^{\mathrm{n}-\mathrm{t}}\left(\mathrm{X}_{\mathrm{t}}-\overline{\mathrm{Z}_{\mathrm{t}}^{\mathrm{T}}} \bar{\beta}\right)\left(\alpha-\mathrm{I}\left[\mathrm{X}_{\mathrm{t}}<\overline{\mathrm{Z}_{\mathrm{t}}^{\mathrm{T}}} \bar{\beta}\right]\right), \lambda \in[0,1]$ is a weighting parameter. The optimization problem can be solved using the methods of linear programming.

It is shown in [23] that CVaR can be estimated as:

$$
\widehat{\operatorname{CVaR}}_{\alpha}^{\mathrm{t}}=\frac{1}{\alpha \mathrm{n}} \sum_{\mathrm{t}=1}^{\mathrm{n}}\left(\mathrm{X}_{\mathrm{t}}-\overline{\mathrm{Z}}_{\mathrm{t}}^{\mathrm{T}} \overline{\beta_{0}}\right)\left(\alpha-\mathrm{I}\left[\mathrm{X}_{\mathrm{t}}<\overline{\mathrm{Z}}_{\mathrm{t}}^{\mathrm{T}} \overline{\beta_{0}}\right]\right)
$$




$$
\widehat{\operatorname{CVaR}}_{\alpha}^{\mathrm{t}}=\frac{1}{\alpha \sum_{\mathrm{t}=1}^{\mathrm{n}} \lambda^{\mathrm{n}-\mathrm{t}}} \sum_{\mathrm{t}=1}^{\mathrm{n}} \lambda^{\mathrm{n}-\mathrm{t}}\left(\mathrm{X}_{\mathrm{t}}-\overline{\mathrm{Z}}_{\mathrm{t}}^{\mathrm{T}} \overline{\beta_{0}}\right)\left(\alpha-\mathrm{I}\left[\mathrm{X}_{\mathrm{t}}<\overline{\mathrm{Z}}_{\mathrm{t}}^{\mathrm{T}} \overline{\beta_{0}}\right]\right) .
$$

\section{Conditional Autoregressive VaR (CaViaR)}

The article [24] proposed to construct VaR autoregressively by analogy with the GARCH models. This model is called CaViaR (conditional autoregressive Value-at-Risk model). Two specifications of $\mathrm{CaViaR}$ model are most popular:

$$
\begin{aligned}
& \operatorname{VaR}_{\alpha}^{\mathrm{t}}=\beta_{0}+\beta_{1} \operatorname{VaR}_{\alpha}^{\mathrm{t}-1}+\beta_{2}\left|\mathrm{X}_{\mathrm{t}-1}\right|-\text { symmetric absolute value model (CaViaRs); } \\
& \operatorname{VaR}_{\alpha}^{\mathrm{t}}=\beta_{0}+\beta_{1} \operatorname{VaR}_{\alpha}^{\mathrm{t}-1}+\beta_{2}\left|\mathrm{X}_{\mathrm{t}-1}\right|+\beta_{3}\left|\mathrm{X}_{\mathrm{t}-1}\right| \mathrm{I}\left[\mathrm{X}_{\mathrm{t}-1}<0\right] \text { - asymmetric slope model (CaViaR). }
\end{aligned}
$$

The estimation of $\beta_{\mathrm{i}}$ can be made via quantile regression.

\section{Experimental Procedures and Results}

This section demonstrates the use of the proposed scheme for the assessment of dynamic risk measures with the determination of the quality of the estimates.

According to the Fig. 2 we follow the next algorithm:

1. The purpose is to obtain the estimates of $\operatorname{VaR}_{\alpha}^{t}$ and $\mathrm{CVaR}_{\alpha}^{\mathrm{t}}$ for the given time series.

2. As the real data we consider two time series of logarithmic return on a daily basis of Asian stock indexes for the period from 2005 to 2015: the oldest and most well-known index of Asian markets Nikkey225 Stock Index (the time series N225_RED) - a composite index of the 225 largest companies publicly traded in Tokyo Stock Exchange, and the composite index, based on 300 stocks trading in the Shanghai stock exchange (the time series CSI300). The Asian stock markets are highly heterogeneous $[6,8]$. N225_RED has a relatively low homogeneous volatility. At the same time, CSI300 demonstrates high volatility. The aim of the N225_RED study is to determine risk measures at a regular market behavior, so we consider data without three time intervals with high volatility of the global financial system: 01.07.2008-01.07.2009, 01.01.2011-01.07.2011, 01.02.2013-01.12.2013.

3. We carry out a general analysis of the studied time series and the time series of variances. With the help of the Ljung-Box test [13] we analyze the dependence of time series members (and their squares) from their previous values. The rejection of null hypothesis (no serial correlation) means the opportunity to use an autoregressive (heteroscedastic) models.

4. We adopt the hypothesis about the possibility of reduction of dynamic risk measures to static. So we may use (3) for the evaluation of $\operatorname{VaR}_{\alpha}^{\mathrm{t}}$ and $\mathrm{CVaR}_{\alpha}^{\mathrm{t}}$. After building the model, it is necessary to confirm or reject this hypothesis. In the latter case, it is necessary to choose an alternative branch in the scheme (Fig. 2).

5. We check the time series for volatility to have the opportunity to use heteroscedastic models. We build a time series of variance by the sliding window method. We choose the window size equal to the half of the sample size and single step. The dependence of the variance from the previous values justifies the use of the heteroscedasticity models for the original time series. We choose the GARCH class of models and determine the order and parameters of the model. We use the built model for $\sigma_{t}$ evaluation.

6. Using $\widehat{\sigma}_{t}$ we construct $\widehat{Z}_{t}=X_{t} / \widehat{\sigma}_{t}$ which are the realizations of the random variable $z_{t}$ (innovations in (2)). Using the BDS-test, the variance ratio test, the Jarque-Bera test, we analyze the properties of $z_{t}$. In accordance with the results, using a classification scheme presented in [19], we choose the method of static $\operatorname{VaR}_{\alpha}(Z)$ and $\mathrm{CVaR}_{\alpha}(\mathrm{Z})$ estimating and get $\widehat{\operatorname{VaR}}_{\alpha}(\mathrm{Z}), \widehat{\mathrm{CVaR}}_{\alpha}(\mathrm{Z})$ estimates.

7. To check the hypothesis about the possibility of reducing of dynamic risk measures to static (step 4) we build a linear regression for empirically determined values of risk measures with respect to the variance estimates (step 5). Using the OLS we determine the linear regression coefficients conduct the regular analysis with the F-test. If the regression equation is essential, the 
$\operatorname{VaR}_{\alpha}^{\mathrm{t}}$ and $\mathrm{CVaR}_{\alpha}^{\mathrm{t}}$ dependence on variances is essential too and the hypothesis is valid. Otherwise, in accordance with Fig. $\mathbf{2}$ it is necessary to use other models (e. g. regressive models).

8. We build dynamic risk measures estimations $\operatorname{VaR}_{\alpha}^{\mathrm{t}}$ and $\operatorname{CVaR}_{\alpha}^{\mathrm{t}}((3))$.

9. We analyze the quality of the dynamic risk measures estimations using the Cupets test $[9,18]$, the Cristoffersen test $[8,18,25]$ and the V-test [18].

\section{1. Risk Measures Estimating for Artificial Data}

To demonstrate and verify the proposed algorithm the artificial data were simulated using $\operatorname{GARCH}(1,1)$ model $((5),(6))$ with parameters $\mathrm{a}_{0}=1, \mathrm{a}_{1}=0.1, \mathrm{~b}_{1}=0.8$ and normally distributed innovations. There is no need to fulfill data analysis and check the hypotheses about the possibility of reducing of dynamic risk measures to static the risk evaluation of dynamic to static measures for artificial data is redundant. The class of the model is also defined. We find the maximum likelihood estimates of the parameters $\hat{\mathrm{a}}_{0}=0.9, \hat{\mathrm{a}}_{1}=0.09, \hat{\mathrm{b}}_{1}=0.81$ and the series of variance estimations $\widehat{\sigma_{t}}$, build the estimations $\widehat{Z}_{t}$ and test them on iid by BDS-test and variance ratio test The result of BDS-test for $\mathrm{m}=4(0.1098<0.9126)$ and the statistic for the variance ratio test $(0.38<1.96)$ confirm that the model residuals are iid.

We use ten the most often used methods, that represent different approaches ([19]), to get $\widehat{\mathrm{VaR}}_{0.9}(\mathrm{Z})$. We compare estimation with the theoretical quantile of normal distribution $\mathrm{VaR} \_$Reall $=1.28$, and the empirical quantile of generated $\mathrm{Z}_{\mathrm{t}}$ (that participated in the construction of artificial data) $\mathrm{VaR}$ _ Real2 $=1.27$ (the relative errors RE1 and RE2 respectively). Using the obtained static assessments $\widehat{\operatorname{VaR}}_{0.9}(\mathrm{Z})$, we build $\operatorname{VaR}_{0.9}^{\mathrm{t}}(\mathrm{Z}) \widehat{\operatorname{VaR}}_{0.9}^{\mathrm{t}}$ evaluations. We analyze the quality of the obtained estimates with the help of the Cupets test (p-value of the statistic LPRpof pv), the test Cristoffersen test ( $p$-value of the statistic LRind pv), and their combination ( $p$-value of the statistic LRec pv) (Table 1).

Table 1

Estimations of static and dynamic VaR for artificial data and the results of the analysis of the estimations

\begin{tabular}{ccccccc}
\hline Method & $\widehat{\operatorname{VaR}}_{0.9}(\mathrm{Z})$ & RE1 & RE2 & LPRpof pv & LRind pv & LRcc pv \\
\hline hist1 & 1.2850 & $-0.27 \%$ & $-1.09 \%$ & 0,894 & 0,937 & 0,988 \\
hist2 & 1.2846 & $-0.24 \%$ & $-1.06 \%$ & 0,406 & 0,515 & 0,573 \\
paramdistr1 & 1.2795 & $0.15 \%$ & $-0.67 \%$ & 1,000 & 0,992 & 1,000 \\
monte_carlo1 & 1.2760 & $0.43 \%$ & $-0.39 \%$ & 1,000 & 0,992 & 1,000 \\
monte_carloR & 1.2777 & $0.30 \%$ & $-0.52 \%$ & 0,714 & 0,671 & 0,854 \\
GEV_quant & 1.2924 & $-0.85 \%$ & $-1.67 \%$ & 0,973 & 0,710 & 0,488 \\
GEV_monte_carlo & 1.2797 & $0.14 \%$ & $-0.68 \%$ & 0,665 & 0,625 & 0,808 \\
GPD_quant & 1.2704 & $0.87 \%$ & $0.06 \%$ & 0,664 & 0,793 & 0,879 \\
GPD_monte_carlo & 1.2717 & $0.76 \%$ & $-0.05 \%$ & 0,506 & 0,992 & 0,986 \\
POT_empirical & 1.2822 & $-0.05 \%$ & $-0.87 \%$ & 0,353 & 0,425 & 0,473
\end{tabular}

We use the following designations in Table 1. The estimation histlwas obtained with the use of the historical simulation method; the Rockafellar - Uryasev discrete method gave the estimation hist2. The evaluation paramdistrl was obtained by explicit formulas under the assumption of a normal distribution with the maximum likelihood estimates of the parameters. Using the Monte Carlo method for estimated parameters of normal distribution, we got the estimation monte_carlo1, the Richardson's method gave the estimation monte_carloR. Use of the theory of EVT yield the following estimates. The evaluation GEV_quant was obtained by explicit formulas using the GEV function with the maximum likelihood estimates of the parameters. Use of the Monte Carlo method for the obtained GEV function gave the estimation GEV_monte_carlo. Similar estimating with the use of GPD function gave the estimates GPD-GPD_quant and GPD_monte_carlo. The assessment POT_emp was obtained by using the empirical POT method.

Using the same methods, we find the $\widehat{\mathrm{CVaR}}_{0.9}(\mathrm{Z})$ estimations and compare them with CVaR_Real1 $=1.755$ found for VaR_Real1 and CVaR_Real2=1.724 found for VaR_Real2 (the rela- 
tive errors RE1 and RE2 respectively). We find $\widehat{\mathrm{CVaR}}_{0.9}^{\mathrm{t}}$ and analyze their quality by using the V-test (Table 2) (statistics $\mathrm{V}_{1}, \mathrm{~V}_{2}, \mathrm{~V}$ ).

Table 2

Estimations of static and dynamic CVaR for artificial data and the results of the analysis of the estimations

\begin{tabular}{ccccccc}
\hline Method & $\widehat{\mathrm{CVaR}}_{0.9}(\mathrm{Z})$ & $\mathbf{R E 1}$ & $\mathbf{R E 2}$ & $\mathbf{V}_{\mathbf{1}}$ & $\mathbf{V}_{\mathbf{2}}$ & $\mathbf{V}$ \\
\hline hist1 & 1,745 & $0,57 \%$ & $-1,23 \%$ & $-0,041$ & $-3,666$ & 1,853 \\
hist2 & 1,745 & $0,57 \%$ & $-1,23 \%$ & 0,025 & $-3,527$ & 1,776 \\
paramdistr1 & 1,753 & $0,11 \%$ & $-1,70 \%$ & $-0,005$ & $-3,627$ & 1,816 \\
monte_carlo1 & 1,747 & $0,48 \%$ & $-1,32 \%$ & $-0,005$ & $-3,627$ & 1,816 \\
monte_carloR & 1,752 & $0,18 \%$ & $-1,63 \%$ & $-0,046$ & $-3,660$ & 1,853 \\
GEV_quant & 1,794 & $-2,22 \%$ & $-4,07 \%$ & $-0,103$ & $-3,717$ & 1,910 \\
GEV_monte_carlo & 1,797 & $-2,37 \%$ & $-4,22 \%$ & $-0,051$ & $-3,663$ & 1,857 \\
GPD_quant & 1,747 & $0,46 \%$ & $-1,35 \%$ & $-0,135$ & $-3,846$ & 1,990 \\
GPD_monte_carlo & 1,748 & $0,40 \%$ & $-1,41 \%$ & $-0,124$ & $-3,910$ & 2,017 \\
POT_empirical & 1,744 & $0,63 \%$ & $-1,17 \%$ & $-0,050$ & $-3,635$ & 1,843
\end{tabular}

The results shown in the tables indicate the correctness of the obtained assessments for static and dynamic risk measures. Fig. 3 demonstrates the modeled dynamic $\operatorname{VaR}_{0.9}^{t}$ and $\mathrm{CVaR}_{0.9}^{\mathrm{t}}$, where the Monte Carlo method for obtained GEV function (GEV_monte_carlo) was used for risk measures model residuals estimating.

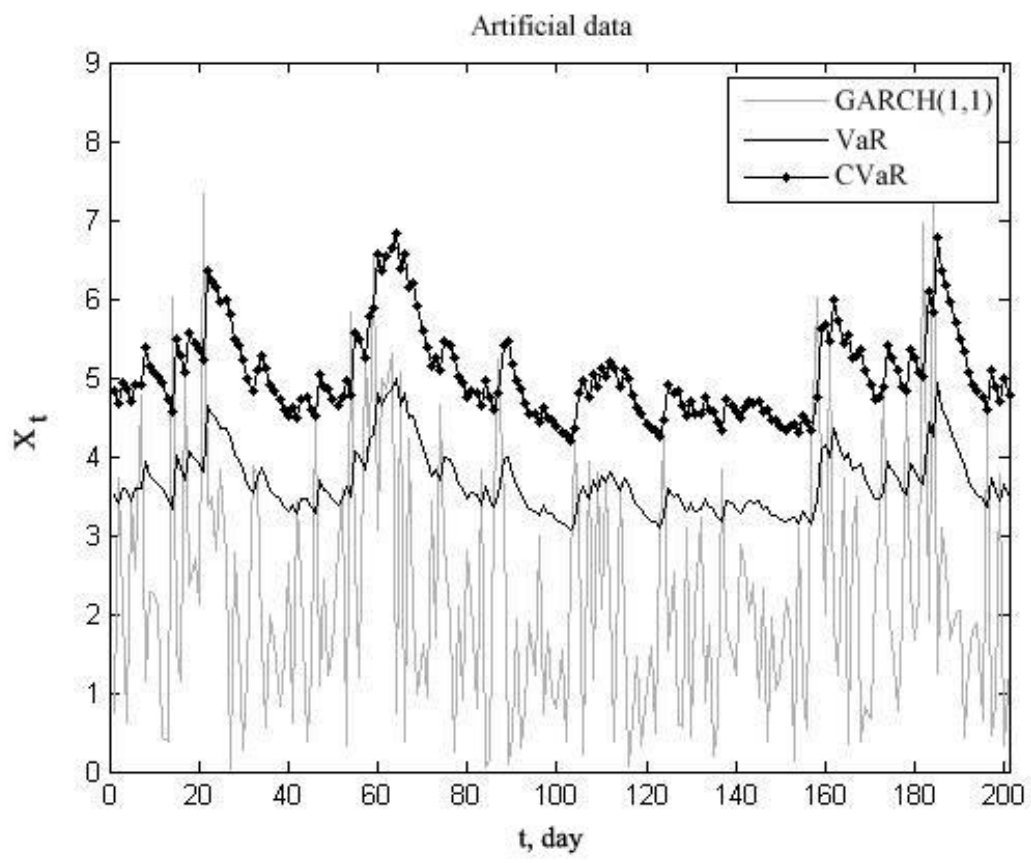

Fig. 3. Modeled dynamic $\operatorname{VaR}_{0.9}^{\mathrm{t}}$ and $\mathrm{CVaR}_{0.9}^{\mathrm{t}}$ for the artificial data, $\mathrm{t}$ - time (day), $\mathrm{X}_{\mathrm{t}}$ - time series

Thus, the analysis leads to the conclusion that the errors of estimations, built by different methods (Fig. 1), are uniform and negligibly small. As is visible from the analysis, the methods, mentioned in the classification scheme (Fig. 1), may be used for risk measures estimating if the starting time series corresponds to the data discussed in this section.

\section{2. Risk Measures Estimating for Real Data}

Table 3 shows the descriptive statistics for the time-series N225_RED $\left(x_{t}\right)$ and the squared series $\left(\mathrm{x}_{\mathrm{t}}^{2}\right)$. As can be seen from Table $\mathbf{6}$, the skewness is close to 0 , and kurtosis is close to 3 , so 
the distribution is close to normal. Ljung-Box test results for $m=7$ ( $Q$ value exceeds the critical value 12.017) show that the main hypothesis is rejected and so there is the dependence of data (and squares) on their previous values.

Table 3

Basic descriptive statistics of the N225_RED

\begin{tabular}{ccccccc}
\hline Statistics & $\mathbf{N}$ & Mean & Std. Deviation & Skewness & Kurtosis & Ljung-Box test \\
\hline $\mathrm{X}_{\mathrm{t}}$ & 1686 & -.0002 & .00414 & .055 & 3.268 & 18.493 \\
$\mathrm{X}_{\mathrm{t}}{ }^{2}$ & 1686 & .0000 & .00003 & 1.557 & 4.372 & 470.667
\end{tabular}

Let's adopt now that the hypothesis about the reduction of dynamic risk measures to static is correct. Build the time series of variance and standard deviations by the sliding window method with a window size 843 and single step, with the Ljung-Box test check the dependence of received series on their previous values. The values of Q-statistics - 5014.942 for the series of variance and 5013.982 for the series of standard deviations are higher than the critical value 10,645 , so the null hypothesis must be rejected. The dependence of the variance (and standard deviations) from their previous values justifies the use of the heteroscedastic models for the time series N225_RED. Since variance can be described as an auto-regression, we may use the models of GARCH class.

To model the time series N225_RED we use GARCH $(3,3)$ model $(4)$ with the parameters $\mathrm{a}_{0}=0, \mathrm{a}_{1}=0.06, \mathrm{a}_{2}=0.08, \mathrm{a}_{3}=0.03, \mathrm{~b}_{1}=0, \mathrm{~b}_{2}=0.48, \mathrm{~b}_{3}=0.26$.

Let's perform the model residuals analysis. The result of BDS-test for $\mathrm{m}=4$ shows that the null hypothesis is accepted (the value of BDS-statistics 0.558 is less than the corresponding quantile of the normal distribution 0.5767 ). The obtained statistic for the variance ratio test also indicates that the model residuals are iid: $0.7477<1.96$. We use the Jarque-Bera test to check the hypothesis about the normal distribution of the residuals. Jarque-Bera statistics is 5.624, which is less than the permissible value equal to 5.649. Therefore, the hypothesis of the normal distribution of the model residuals should be adopted.

To confirm the hypothesis about the possibility of using the static risk measure in the calculation of the dynamic risk measures, we build a linear regression (8). Using OLS, we estimate the linear regression coefficients $\mathrm{a}_{1}, \mathrm{a}_{2}$ and use the F-test to check the significance of the linear regression. The value of the F-statistic is equal to 18324.548 and exceeds the critical value, the value of the coefficient of determination is equal to 0.956 , so we may conclude that the regression is significant. Thus, we can use the model (8) to construct a model of dynamic risk measures.

Taking into account the previous analysis, five of ten methods that have been used for the artificial data are considered. Table 4 presents the $\mathrm{VaR}_{0.95}$ estimations and the p-values of quality tests.

Table 4

The $\mathrm{VaR}_{0.95}$ estimations of the model residuals for the N225_RED and the p-values of quality tests

\begin{tabular}{ccccc}
\hline Method & VaR $_{\mathbf{0 . 9 5}}$ & LRpof pvalue & LRind pvalue & LRcc pvalue \\
\hline hist1 & 1,902 & 0,018 & 0,073 & 0,012 \\
paramdistr1 & 1,703 & 0,186 & 0,053 & 0,064 \\
GEV_quant & 1,826 & 0,044 & 0,021 & 0,009 \\
GPD_quant & 1,762 & 0,135 & 0,043 & 0,042 \\
POT_empirical & 1,172 & 0,000 & 0,024 & 0,000
\end{tabular}

In contrast to the results of section 3.1, the values of the quality criteria are not homogeneous: the tests give a positive result (statistic value must be more than the chosen confidence level 0.05 ) only in the case of paramdistrl method. Thus, it is confirmed that the residuals distribution is close to normal and for VaR estimation the explicit formulas for normal distribution of innovations with maximum likelihood estimates of the parameters should be used. 
Table 5 presents the $\mathrm{CVaR}_{0.95}$ estimations, found with the same methods and their quality characteristics. Analysis of the results also leads to the conclusion that the paramdistrl method should be used.

\section{Table 5}

The $\mathrm{CVaR}_{0.5}$ estimations of model residuals for the N_225 index and the results of quality tests

\begin{tabular}{ccccc}
\hline Method & $\mathbf{C V a R}_{\mathbf{0 . 9 5}}$ & $\mathbf{V}_{\mathbf{1}}$ & $\mathbf{V}_{\mathbf{2}}$ & $\mathbf{V}$ \\
\hline hist1 & 2,233 & 0,0170 & $-0,0001$ & 0,0086 \\
paramdistr1 & 2,126 & 0,0166 & 0,0007 & 0,0087 \\
GEV_quant & 2,401 & 0,0167 & 0,0011 & 0,0088 \\
GPD_quant & 2,319 & 0,0174 & $-0,0024$ & 0,0099 \\
POT_empirical & 1,782 & 0,0173 & $-0,0038$ & 0,0106
\end{tabular}

Fig. 4 shows the modeled $\mathrm{VaR}_{0.95}^{\mathrm{t}}$ and $\mathrm{CVaR}_{0.95}^{\mathrm{t}}$ for this time series, where the risk measures estimations were obtained by the explicit formulas for normal distribution of innovations with maximum likelihood estimates of the parameters (paramdistr1).

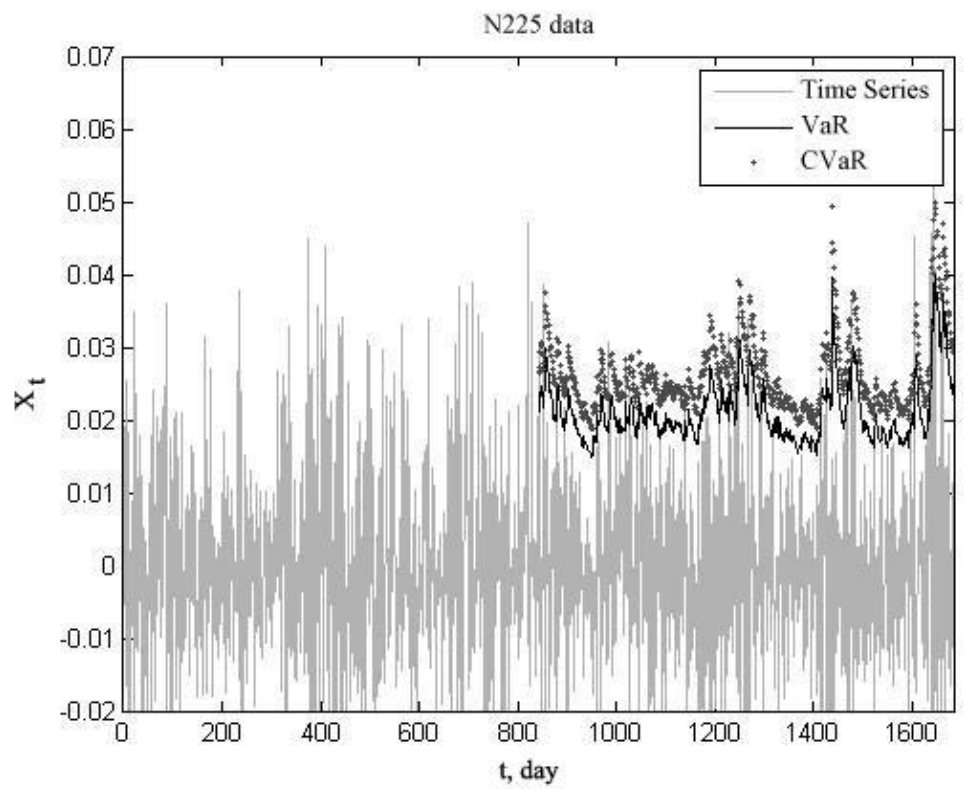

Fig. 4. Modeled dynamic $\mathrm{VaR}_{0.95}^{\mathrm{t}}$ and $\mathrm{CVaR}_{0.95}^{\mathrm{t}}$ estimates for the $\mathrm{N} 225$ index, $\mathrm{t}$ - time (day), $\mathrm{X}_{\mathrm{t}}$ - time series

A similar analysis was carried out for the time-series CSI300. Table 6 shows the descriptive statistics for the time series and squared series and the results of the Ljung-Box test for $\mathrm{m}=7$.

Table 6

Basic descriptive statistics of the CSI300

\begin{tabular}{ccccccc}
\hline Statistics & $\mathbf{N}$ & Mean & Std. Deviation & Skewness & Kurtosis & Ljung-Box test \\
\hline $\mathrm{X}_{\mathrm{t}}$ & 2445 & -.0002 & .00393 & .186 & 3.268 & 22.754 \\
$\mathrm{X}_{\mathrm{t}}{ }^{2}$ & 2455 & .0000 & .00003 & 1.754 & 4.372 & 431.487
\end{tabular}

Ljung-Box test indicates the data (and squared data) dependence on their previous values.

Let's adopt that the hypothesis about the reduction of dynamic risk measures to static is correct. Let's build the time series of variance and standard deviations by the sliding window with a window size 1222 and single step, with the Ljung-Box test check the dependence of received 
series on their previous values. The values of Q-statistics - 8585.195 for the series of variance and 8583.195 for the series of standard deviations are higher than the critical value 12.017 , so the null hypothesis about the independence must be rejected.

To model the time series CSI300 the $\operatorname{GARCH}(5,0)$ model (4) with parameters $\mathrm{a}_{0}=0.00014$, $\mathrm{a}_{1}=0.0713, \mathrm{a}_{2}=0.15300, \mathrm{a}_{3}=0.12165, \mathrm{a}_{4}=0.18222, \mathrm{a}_{5}=0.10322$ was used.

Perform the model residuals analysis. The BDS-statistic for $\mathrm{m}=3$ is 0.13975 that is less than the corresponding quantile of the normal distribution 0.5767 so the null hypothesis is accepted. The statistic for the variance ratio test also confirms that the model residuals are iid $(1.6815<1.96)$. Using Jarque-Bera test, we reject the hypothesis about the normal distribution of the residuals $(267.19>5.968)$.

To confirm the hypothesis about the reduction of dynamic risk measures to static we build a linear regression (8) and conduct the F-test. The value of the F-statistic (46479.676) and the value of the coefficient of determination (0.974) show that the regression is significant.

We use the same five methods to estimate risk measures for the model residuals. The results of evaluation are shown in Tables 7, 8.

Table 7

The $\operatorname{VaR}_{0.9}$ estimation of the model residuals for the CSI300 and the p-values of quality tests

\begin{tabular}{ccccc}
\hline Method & VaR $_{\mathbf{0 . 9}}$ & LRpof pvalue & LRind pvalue & LRcc pvalue \\
\hline hist1 & 1,315 & 0,000 & 0,465 & 0,000 \\
paramdistr1 & 1,334 & 0,000 & 0,291 & 0,000 \\
GEV_quant & 1,271 & 0,001 & 0,775 & 0,002 \\
GPD_quant & 1,187 & 0,156 & 0,951 & 0,054 \\
POT_empirical & 1,012 & 0,284 & 0,846 & 0,553
\end{tabular}

Table 8

The $\mathrm{CVaR}_{0.9}$ estimations of the model residuals for CSI300 and the results of quality tests

\begin{tabular}{ccccc}
\hline Method & $\mathbf{C V a R}_{\mathbf{0 . 9}}$ & $\mathbf{V}_{\mathbf{1}}$ & $\mathbf{V}_{\mathbf{2}}$ & $\mathbf{V}$ \\
\hline VaR_hist1 & 1,059 & 0,0193 & 0,0016 & 0,0104 \\
VaR_paramdistrl & 1,837 & 0,0051 & $-0,0015$ & 0,0033 \\
VaR_GEV_quant & 2,028 & 0,0042 & $-0,0008$ & 0,0025 \\
VaR_GPD_quant & 1,922 & 0,0026 & $-0,0010$ & 0,0018 \\
VaR_POT_empirical & 1,403 & 0,017 & $-0,0013$ & 0,0065
\end{tabular}

Analyzing the results we may conclude that the methods GPD_quant and GEV_quant (explicit formulas for estimating using the GPD function and GEV function respectively with maximum likelihood estimates of the parameters) provides estimates that satisfy the quality criteria. So, the models with normal distributed innovations cannot be used for risk measures calculating. Innovations exhibit heavy tails, and, in accordance with the classification scheme (Fig. 1), the POT models, using the GPD function or GEV function, should be used. Fig. $\mathbf{5}$ shows the modeled $\mathrm{VaR}_{0.9}^{\mathrm{t}}$ and $\mathrm{CVaR}_{0.9}^{\mathrm{t}}$ for this time series.

Fig. 4, 5 show the values of the time series with estimates of dynamic risk measures VaR and CVaR. It is seen from the figures, that estimates display positive boundary changes of time series in time very well. 


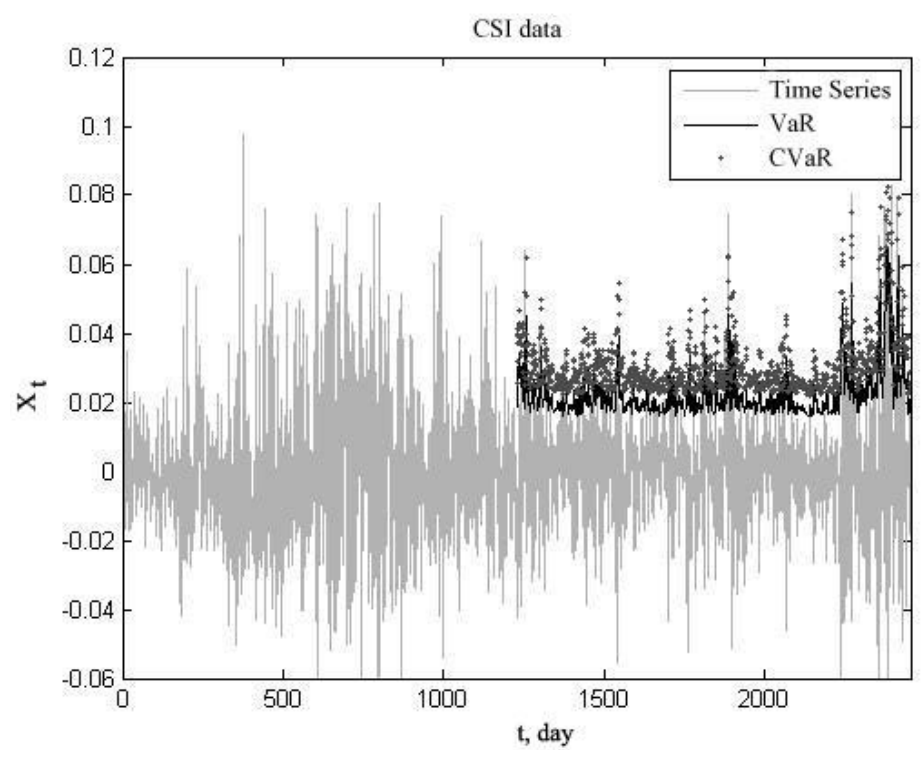

Fig. 5. Modeled dynamic $\operatorname{VaR}_{0.9}^{\mathrm{t}}$ and $\mathrm{CVaR}_{0.9}$ estimates for the CSI300, $\mathrm{t}$ - time (day), $\mathrm{X}_{\mathrm{t}}$ - time series

\section{Discussion of the obtained risk measures estimates}

The analysis of the results for artificial data (Table 1, 2, Fig. 3) leads to the conclusion that the errors of estimations, built by different methods from Fig. 1, are uniform and negligibly small. The methods, mentioned in the classification scheme (Fig. 1), may be used for risk measures estimating if the time series corresponds to the data discussed in this section.

The analysis of the real time series using the classification scheme Fig. 2 leads to the following choice for dynamic VaR and CVaR estimating. The residuals of model for the time series Nikkey225StockIndex demonstrate the distribution close to normal and so the explicit formulas for normal distribution with maximum likelihood estimates of the parameters should be used (Table 3-5, Fig. 4). At the same time, POT models are the most convenient for CSI300 because the residuals demonstrate heavy tails (Table 6-8, Fig. 5).

As follows from the above examples, the application of the classification scheme simplifies the choice of the method of dynamic risk measures estimation. The proposed selection procedure allows a specialist without a deep knowledge of mathematical statistics unit to evaluate dynamic VaR and CVaR. At the same time, in this study we did not aim to describe and apply all existing methods for dynamic VaR and CVaR estimating. Therefore, of course, the proposed scheme may be supplemented by new methods for assessments and new tests to assess their quality.

\section{Conclusions}

The most popular methods of dynamic risk measures estimating VaR, CVaR have been discussed in this paper, the description of the methods have been carried out, the advantages and disadvantages of the methods and conditions of their application have been analyzed The systematization and classification of methods have been proposed. The systematization of estimation methods provides the user the ordered set of research methods and facilitates the search and analysis of suitable procedures for risk measures estimating. The proposed classification table simplifies the choice of estimation method, reducing the selection process to the implementation and analysis of results of system of tests.

Application of the classification scheme, as well as its effectiveness, is illustrated by the dynamic risk measures VaR, CVaR estimation for artificially generated series and two time series of logarithmic return on a daily basis of the most well-known Asian stock indexes Nikkey225StockIndex and CSI300.

The proposed qualification scheme may be useful for risk managers from various financial institutions, who have a purpose to evaluate the risks of the stock markets. Active development of 
this direction in recent years leads to the continuous development of new methods and approaches, which consequently leads to the need to make additions and amendments to the proposed scheme.

\section{References}

[1] Morgan, J. P. (1996). Risk Metrics. Technical Document, $4^{\text {th }}$ edition. New York, 284.

[2] Artzner, P., Delbaen, F., Eber, J.-M., Heath, D. (1999). Coherent Measures of Risk. Mathematical Finance, 9 (3), 203-228. doi: 10.1111/1467-9965.00068

[3] Chun, S. Y., Shapiro, A., Uryasev, S. (2012). Conditional Value-at-Risk and Average Valueat-Risk: Estimation and Asymptotics. Operations Research, 60 (4), 739-756. doi: 10.1287/opre.1120.1072

[4] Filer, R. K., Žiković, S. (2013). Ranking of VaR and ES Models: Performance in Developed and Emerging Markets. Finance a úvěr-Czech Journal of Economics and Finance, 4 (63), 327-359.

[5] Nadarajah, S., Zhang, B., Chan, S. (2013). Estimation methods for expected shortfall. Quantitative Finance, 14 (2), 271-291. doi: 10.1080/14697688.2013.816767

[6] Bao, Y., Lee, T.-H., Saltoglu, B. (2006). Evaluating predictive performance of value-at-risk models in emerging markets: a reality check. Journal of Forecasting, 25 (2), 101-128. doi: 10.1002/for.977

[7] Guégan, D., Zhao, X. (2013). Alternative modeling for long term risk. Quantitative Finance, 14 (12), 2237-2253. doi:10.1080/14697688.2013.835860

[8] Yoon, S. M., Kang, S. H. (2013). VaR Analysis for the Shanghai Stock Market. The Macrotheme Review, 2 (6), 89-95.

[9] Karadzic, V., Cerovic, J. (2014). Market risk of the Western Balkans countries during the global financial crisis. Economic Annals-XXI, 19-24.

[10] Magadia, J. (2011). Confidence interval for expected shortfall using bootstrap methods. 4th Annual BSP UP Professorial Chair Lectures, 21-23.

[11] Chen, S. X. (2007). Nonparametric Estimation of Expected Shortfall. Journal of Financial Econometrics, 6 (1), 87-107. doi: 10.1093/jjfinec/nbm019

[12] Zgurovskyj, M. Z., Pankratova, N. D. (2007). Osnovy systemnogo analizu. Kyiv: BHV, 544.

[13] Tsay, R. S. (2010). Analysis of Financial Time Series. (third edition). Hoboken: John Wiley Sons, Inc., 712 .

[14] Bidyuk, P. I., Romanenko, V. D., Tymoshhuk, O. L. (2013). Analiz chasovyh ryadiv. Kyiv: Politehnika, NTUU «KPI», 601.

[15] Embrechts, P., Kaufmann, R., Patie, P. (2005). Strategic Long-Term Financial Risks: Single Risk Factors. Comput Optim Applic, 32 (1-2), 61-90. doi: 10.1007/s10589-005-2054-7

[16] Fiszeder, P., Orzeszko, Wi. (2012). Nonparametric Verification of GARCH-Class Models for Selected Polish Exchange Rates and Stock Indices. Finance a úvěr-Czech Journal of Economics and Finance, 5 (62), 430-449.

[17] Cheong, C. W. (2009). A variance ratio test of random walk inenergy spot markets. Journal of quantitative economics, 1 (8), 106-117.

[18] Kjellson, B. (2013). Forecasting Expected Shortfall. An Extreme Value Approach. Bachelor's thesis in Mathematical Sciences, 43.

[19] Zrazhevskaja, N. G., Zrazhevskij, A. G. (2016). Klassifikacija mer riska dlja odnoj sluchajnoj velichiny. Systemni doslidzhennya ta informacijni texnologiyi, 2.

[20] Pankratova, N. D., Zrazhevskaja, N. G. (2015). Model' avtokorreljacionnoj funkcii vremennogo rjada s sil'noj zavisimost'ju. Problemy upravlenija i informatiki, 5, 102-112.

[21] González-Rivera, G., Lee, T.-H., Yoldas, E. (2007). Optimality of the RiskMetrics VaR model. Finance Research Letters, 4 (3), 137-145. doi: 10.1016/j.frl.2007.06.001

[22] McMillan, D. G., Kambouroudis, D. (2009). Are RiskMetrics forecasts good enough? Evidence from 31 stock markets. International Review of Financial Analysis, 18 (3), 117-124. doi:10.1016/ j.irfa.2009.03.006

[23] Taylor, J. W. (2008). Using Exponentially Weighted Quantile Regression to Estimate Value at Risk and Expected Shortfall. Journal of Financial Econometrics, 6 (3), 382-406. doi:10.1093/jjfinec/nbn007

[24] Engle, R. F., Magnelli, S. (1999). CaViaR: Conditional Autoregressive Value at Risk by Quantile Regression. NBER: Working paper, 7341.

[25] Dowd, K. (2005). Measuring Market Risk (second edition). Chichester: John Wiley and Sons. doi: $10.1002 / 9781118673485$ 